\title{
BMJ Open Utility of a personalised Bronchiectasis Action Management Plan (BAMP) for children with bronchiectasis: protocol for a multicentre, double-blind parallel, superiority randomised controlled trial
}

Julie M Marchant (D) , ${ }^{1,2}$ Anne B Chang, ${ }^{1,2,3,4}$ Kobi L Schutz, ${ }^{3,5}$ Lesley Versteegh, ${ }^{3}$ Anne Cook, ${ }^{1}$ Jack Roberts, ${ }^{1}$ Peter S Morris, ${ }^{3}$ Stephanie T Yerkovich, ${ }^{1,3}$ Gabrielle B McCallum ${ }^{3}$

To cite: Marchant JM, Chang $A B$, Schutz KL, et al. Utility of a personalised Bronchiectasis Action Management Plan (BAMP) for children with bronchiectasis: protocol for a multicentre, double-blind parallel, superiority randomised controlled trial. BMJ Open 2021;11:e049007. doi:10.1136/ bmjopen-2021-049007

- Prepublication history for this paper is available online. To view these files, please visit the journal online (http://dx.doi org/10.1136/bmjopen-2021049007).

Received 12 January 2021 Accepted 15 November 2021

Check for updates

(C) Author(s) (or their employer(s)) 2021. Re-use permitted under CC BY-NC. No commercial re-use. See rights and permissions. Published by BMJ.

For numbered affiliations see end of article.

Correspondence to

Dr Julie M Marchant;

jm.marchant@qut.edu.au

\section{ABSTRACT}

Introduction Bronchiectasis is no longer considered rare or irreversible in children, yet it remains relatively under-researched and neglected in respiratory health globally. Bronchiectasis (including chronic suppurative lung disease) causes substantial morbidity for patients and significant impact on caregivers, especially during acute respiratory exacerbations. In other chronic respiratory diseases (eg, asthma), empowering consumers with an individualised plan for management of acute exacerbations improves clinical outcomes. However, in the absence of any such data specific to bronchiectasis, action management plans are rarely currently used in children or adults with bronchiectasis. We hypothesise that providing an individualised bronchiectasis action management plan (BAMP) to children with bronchiectasis reduces nonscheduled doctor consultations, compared with not having a BAMP.

Methods and analysis This multicentre, parallel, double-blind, randomised trial involving three urban Australian hospitals commenced in June 2018 and will include 198 children, aged $<19$ years with bronchiectasis who had 2 or more exacerbations in the previous 18 months. Children will be randomised to having an individualised BAMP or standard care (a decoy clinic letter). Primary caregivers will then be followed up monthly for 12 months. The primary outcome is the rate of acute non-scheduled doctor visits for respiratory exacerbations by 12 months. The main secondary outcomes are cough-specific quality of life scores at 6 and 12 months, overall exacerbation rate over 12 months, and proportion of children who received timely influenza vaccination by 30 May annually.

Ethics and dissemination The Human Research Ethics Committees of the Northern Territory Department of Health and Menzies School of Heath Research and Queensland Children's Hospital approved the study. The results of the trial will be submitted for publication and the BAMP made available free online.

Trial registration number Australia and New Zealand Clinical Trials Register ACTRN12618000604202.
Strengths and limitations of this study

- The first such study addressing a clinical need/priority and knowledge gap identified by primary caregivers of children with bronchiectasis.

- Our multicentre, parallel, double-blind, randomised trial will provide important new information on whether the routine provision of an individualised written bronchiectasis action management plan (BAMP), compared with standard care, reduces non-scheduled doctor visits for children with bronchiectasis.

- We are evaluating the efficacy of provision of a BAMP on patient-informed outcomes (quality of life and exacerbations) as well as timely annual influenza vaccination.

- While we have a decoy control group, we cannot be certain that the parents and persons collecting the outcome data will remain blinded to the child's allocation group throughout the study.

- The current SARS-CoV-2 pandemic has resulted in a substantial fall in acute respiratory exacerbations (our primary outcome), which is likely to result in an inadequate sample size which was calculated based on patient data before the pandemic.

\section{INTRODUCTION}

Bronchiectasis (including chronic suppurative lung disease) unrelated to cystic fibrosis (CF) remains a major contributor to chronic lung morbidity ${ }^{12}$ with a high unmet need globally. ${ }^{3}{ }^{4}$ International registries, such as European Multicentre Bronchiectasis Audit and Research Collaboration, have highlighted this increasingly recognised global health issue. ${ }^{5}$ Prevalence studies in adults in the UK (20\% increase from 2008 to 2012) ${ }^{6}$ and USA (increased $8.7 \%$ per year between 2000 and 2007) ${ }^{7}$ have shown the increasing burden of bronchiectasis on healthcare 
systems. In New Zealand, Munro et al found a $280 \%$ increase in the incidence of children treated in bronchiectasis clinics between 2000 and 2008. ${ }^{8}$ In Australia, while it is known that the prevalence of bronchiectasis among Australian First Nations children is very high (one in every 68), ${ }^{9}$ its burden among non-First Nations children is underappreciated. ${ }^{10}$ Indeed, an Australianwide study found that $\sim 10 \%$ of 346 children newly referred to respiratory specialist for chronic $\operatorname{cough}^{11}$ had bronchiectasis.

Deemed by the European Respiratory Society (ERS) as one of the most neglected lung disorders ${ }^{12}$ with a high patient burden, needs and cost, ${ }^{2}{ }^{13}$ research addressing patient needs and priorities are required urgently. Further, children with bronchiectasis unrelated to $\mathrm{CF}$ is underserviced compared with other chronic respiratory disease. ${ }^{14}$ There are few resources and relatively little research relevant to paediatric bronchiectasis, ${ }^{1}$ in contrast to chronic diseases like CF. In addition, despite once considered invariably progressive, it is now accepted that bronchiectasis disease progression can be halted and/or reversed if treatment is early and management is optimised. ${ }^{1}$ Thus, optimisation of clinical care in children with bronchiectasis has major longterm patient benefits (eg, reducing disease severity) ${ }^{1}$ as well as socioeconomic benefits for healthcare systems worldwide.

For people with bronchiectasis, recurrent acute respiratory exacerbations or flare-ups are common, with some requiring hospitalisation. ${ }^{10}$ Published data show that respiratory exacerbations have major negative health impacts on people with underlying lung diseases. ${ }^{15} 16$ For example, in children with bronchiectasis, respiratory exacerbations are particularly important clinically, as they are associated with increased psychological stress, impaired quality of life (QoL), lung function decline ( -1.9 forced expiratory volume in one second $\left(\mathrm{FEV}_{1}\right) \%$ predicted per hospitalised exacerbation) and substantial healthcare costs. ${ }^{13} 17$ A recent study further demonstrated the psychosocial impact of exacerbations with high rates of school absences (24.9 children per 100 child months) and parental time off work for children with bronchiectasis. ${ }^{18}$ Further, in Australia, each paediatric hospitalisation for bronchiectasis costs $\sim$ A33 $000^{13}$ highlighting the economic cost. A systematic review of adult economic burden similarly reflects hospitalisations are a major economic cost driver worldwide. ${ }^{19}$ Thus, addressing ways to reduce respiratory exacerbations and/or the severity of exacerbations is important to improve clinical and socioeconomic outcomes in bronchiectasis.

Further, respiratory exacerbations are also very important from the consumer perspective. ${ }^{20}$ In the European Lung Foundation led survey ${ }^{21}$ undertaken by 225 respondents (parents of children with bronchiectasis and young adults with childhood bronchiectasis) from 21 countries, having an action management plan for flare-ups/ exacerbations was one of their top-ranked clinical priority to improve self-management. ${ }^{21}$ Thus, addressing respiratory exacerbations is not only important from the medical perspective but also aligns with priorities expressed by parents of children with bronchiectasis, ${ }^{21}$ as well as the research priorities of the $\mathrm{ERS}^{20}$ and $\mathrm{USA}^{4}$ adults with bronchiectasis.

In children with asthma, personalised asthma action plans are recommended in all settings, as there is robust evidence that its use improves clinical outcomes (eg, reduced acute doctor visits, hospitalisations and improved QoL). ${ }^{22}$ Therefore, using an action plan for children with bronchiectasis may also be efficacious in improving these clinical outcomes. However, it is currently unknown if use of a personalised bronchiectasis action management plan (BAMP) is also beneficial for people with bronchiectasis. To date, there are no such studies in children or adults with bronchiectasis. ${ }^{2324}$ A recent systematic review specific to the use of an action plan for bronchiectasis in children confirmed the absence of randomised controlled trials (RCTs). ${ }^{23}$ A Cochrane review on self-management in bronchiectasis further highlighted the paucity of evidence in both adults and children, ${ }^{24}$ finding only two small poor quality trials in adults using interventions of education and/or rehabilitation, neither with an action plan intervention and highlighted the need for research in children. With this major research gap of whether using a personalised BAMP improves clinical outcomes, it is not surprising that a BAMP is not used routinely, even in tertiary centres, when managing children with bronchiectasis. Providing each patient with a personalised BAMP adds substantial time to routine clinical consultations which is an issue as doctors are increasingly asked to do more with less available clinical time. Thus, an efficacy trial is needed before a BAMP can be successfully implemented in routine clinical care.

\section{Study objectives and hypotheses}

Our primary question is to determine if the routine use of a personalised written BAMP, compared with standard care, reduces non-scheduled doctor visits for children with bronchiectasis. Our primary hypothesis is that providing an individualised BAMP to children with bronchiectasis reduces non-scheduled doctor consultations, compared with not having a BAMP.

Our secondary aims are to evaluate if the routine use of a personalised BAMP (compared with standard care):

1. reduces the overall rate of acute exacerbation over the intervention period (12 months);

2. improves cough-specific QoL (PC-QoL) ${ }^{25}$ at 6 and 12 months; and

3. improves early uptake of annual influenza vaccine (by 30 May annually).

Our secondary hypotheses are the provision a BAMP, compared with standard care, will result in a lower rate of respiratory exacerbations, improved PC-QoL and higher rate of timely annual influenza vaccination.

\section{METHODS AND ANALYSIS}

\section{Study setting and design}

We are conducting a multicentre, parallel group, superiority RCT design (with concealed allocation 1:1) undertaken in Darwin (Northern Territory), Brisbane 


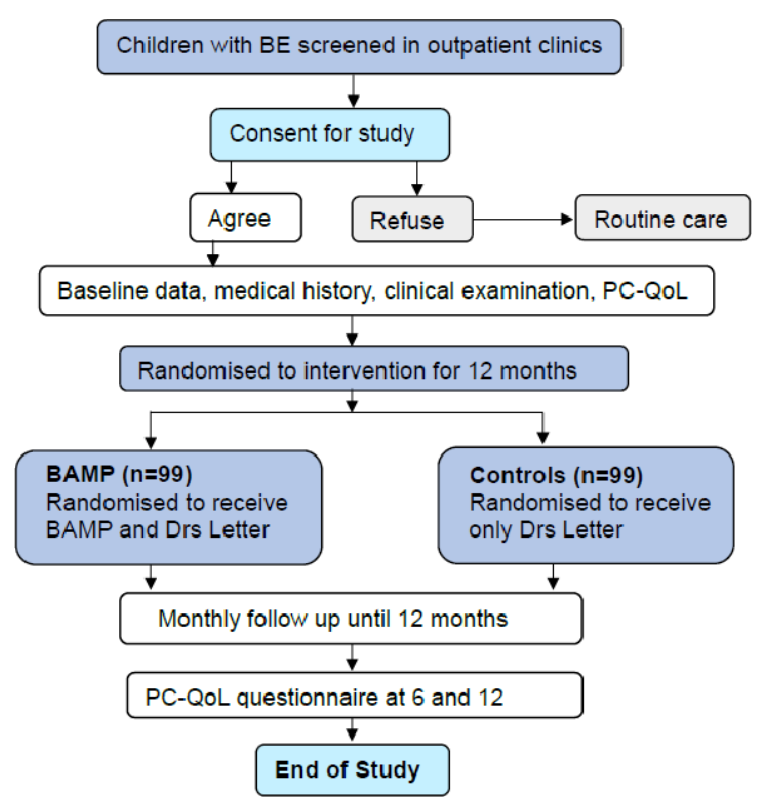

Figure 1 Schematic study design.

(Queensland) and Perth (Western Australia). Our study design, summarised in figure 1 , is in accordance with the recommendations for the Interventional Trial (Standard Protocol Items: Recommendations for Interventional Trials) guidelines. ${ }^{26}$ Our study is ongoing; we recruited our first participant on 30 June 2018.

\section{Study population}

The inclusion criteria are: (1) children aged $<19$ years with bronchiectasis or chronic suppurative lung disease, (2) had $\geq 2$ non-scheduled doctor visits or respiratory exacerbations in the previous 18 months, and (3) do not currently have a BAMP. Our exclusion criteria are: (1) children with CF, (2) children who have an existing written plan, or (3) inability to follow-up the children for 12 months (eg, no mobile phone).

\section{Recruitment, randomisation and allocation}

Primary caregivers of eligible children are approached in outpatient clinics of the study sites by trained research nurses (Royal Darwin Hospital, Queensland Children's Hospital, Perth Children's Hospital) with study information and informed consent gained. After written informed consent, children will be randomised (1:1 allocation) to receive either (1) standard care (control group); or (2) a written pictorial based BAMP (intervention group). A computer-generated permuted block randomisation sequence (permuted block sizes of 4-8), will be prepared by a statistician external to the study team. Randomisation is stratified by site (Brisbane, Darwin, Perth), age ( $\leq 12$ or $\geq 12$ years) and type of patient (recent diagnosis ( $<12$ months) or later ( $\geq 12$ months)). Treatment groups are allocated according to the randomisation number concealed as per our previously completed National Health and Medical Research Council-funded RCTs ${ }^{27-29}$ and will not be revealed until data analyses are complete.

\section{Procedures, intervention and controls}

At enrolment, the primary caregiver of each child is informed by the research nurse that they will receive some sort of a management plan via standard mail (and email where available). They are unaware of the format of the management plan.

The primary respiratory physician will complete a personalised BAMP (action plan link www.improvebe. org/news-resources) for each child after the clinic visit, in addition to dictating the usual clinic letter for the child's family doctor. The individualised BAMP is then given to the study's unblinded respiratory physician (JM) who standardises the BAMP (typed) and ensures that the child's BAMP is correct. The BAMP front page consists of the child's demographics and key data on the child's bronchiectasis (aetiology, date of CT scan and location of bronchiectasis, latest airway microbiology results) and when their annual influenza vaccine is next due. The second page consists consist of several key points: (1) what the child's daily treatment regimen is (eg, type of medication(s) and frequency, airway clearance technique), (2) what to do when there is a flare up (including medications) and (3) indications when to see a doctor. The action plan has three different coloured sections: baseline (in green), exacerbation (orange) and non-resolution of an exacerbation or severe exacerbation (red). The green section includes data on the child's baseline clinical state (eg, no daily cough and sputum), his/her routine treatment (medications (if any) and airway clearance technique). The orange zone outlines the symptoms of an exacerbation for the child (eg, cough increase, sputum production, wheeze, shortness of breath, reduced physical activity or appetite) and what to do during an exacerbation (medications [dose, frequency and duration] and airway clearance techniques). The red zone outlines when to see a doctor or present to hospital (eg, worsening or persistent cough despite oral antibiotics, increased work of breathing, unable to take part in normal activity).

The unblinded physician will send the BAMP and the doctors clinic letter to primary caregivers of children allocated to the intervention arm. This will be accompanied by a cover letter explaining the BAMP and how they can use it, that is, they can show the BAMP to any general practitioners, community clinics, emergency departments or school/day care they attend, but not to show it to their respiratory physician or the study research nurses. They have email and phone access to the unblinded physician to clarify any section of the BAMP.

For children in the control arm, the doctors clinic letter serves as the decoy management plan. A similar cover letter accompanied the decoy letter and they also had email and phone access to the unblinded physician to clarify anything in the letter.

The child's allocation will be blinded to the child's respiratory physician and the primary caregivers who will be unaware that the BAMP is the intervention component. Research nurses also remain blinded to the child's allocation when collecting outcome data. At the end of 
Table 1 Timeline of procedures and follow-up

\begin{tabular}{|c|c|c|c|c|c|c|c|}
\hline & \multirow{2}{*}{$\begin{array}{l}\text { All } \\
\text { Baseline } \\
\text { visit }\end{array}$} & \multicolumn{2}{|c|}{ Allocated groups } & \multicolumn{4}{|c|}{ All } \\
\hline & & Intervention & Control & $\begin{array}{l}\text { Monthly for } \\
12 \text { months }\end{array}$ & 6 months & 12 months & 13 months \\
\hline $\begin{array}{l}\text { Written informed } \\
\text { consent }\end{array}$ & $\sqrt{ }$ & & & & & & \\
\hline Randomised & $\sqrt{ }$ & & & & & & \\
\hline $\begin{array}{l}\text { Medical history and } \\
\text { chart review }\end{array}$ & $\sqrt{ }$ & & & & & & \\
\hline Clinical assessment & $\sqrt{ }$ & & & & & & \\
\hline PC-QoL-8 & $\sqrt{ }$ & & & & $\sqrt{ }$ & $\sqrt{ }$ & \\
\hline $\begin{array}{l}\text { BAMP written by } \\
\text { child's doctor }\end{array}$ & $\sqrt{ }$ & & & & & & \\
\hline $\begin{array}{l}\text { BAMP given by study } \\
\text { doctor }\end{array}$ & & $\sqrt{ }$ & & & & & \\
\hline Revised BAMP given & & & & & & & $\sqrt{ }$ \\
\hline
\end{tabular}

BAMP, bronchiectasis action management plan; PC-QoL-8, parent-proxy chronic cough quality of life.

the study (month 13), the unblinded respiratory physician sends a current individualised BAMP to all participants in the study.

\section{Data collection}

An overview and timeline of procedures and follow-up is summarised in table 1 .

Data are collected from the primary caregiver and from the medical records at baseline (enrolment). Caregivers are asked to complete a validated parent-proxy chronic cough quality of life (PC-QoL-8) ${ }^{25}$ questionnaire at baseline, 6 and 12 months. Events, including exacerbations and doctor visits, will be monitored by research assistants who will keep monthly contact with the parents, as per our previous RCTs on bronchiectasis. ${ }^{27} 28$ Exit point of the study will be caregiver or physician requesting for the child to be withdrawn.

At enrolment, demographic information (age, sex, ethnicity, household size, etc), birth history, breastfeeding history, prior illness, immunisation record, in utero and household smoke exposure are recorded, and a physical examination performed. Immunisation data are collected from the Australian immunisation register or patients' records. All data are documented on hard copy standardised forms and entered into the database later. Deidentified data in the central database is password protected and confidentiality maintained.

\section{Outcome measures}

Events are being collected from active surveillance of participants and electronic records, defined using our established methods. ${ }^{27} 28{ }^{30}$ Research nurses collecting outcome data are blinded to allocation groups. Our primary outcome is the difference between groups in the rate of acute doctor visits for respiratory exacerbations.
Secondary outcomes are: (1) overall respiratory exacerbations rate over 12 months; (2) PC-QoL ${ }^{25}$ scores at 6 and 12 months; and (3) proportion who received influenza vaccination by 30 May annually.

Respiratory exacerbations is defined as an acute respiratory episode that: (1) is treated with antibiotics and (2) an increase in sputum volume or purulence, or $\geq 3$ days of change in cough $\left(>20 \%\right.$ increase in cough score ${ }^{31}$ or type (dry to wet/productive)) or physician confirmed acute change in respiratory rate, work of breathing or chest signs. ${ }^{32}$ We are using the PC-QoL- $8,{ }^{25}$ a validated parentproxy cough-specific QoL that consist of 8 questions with a summary score between 1 (most impact that is, poorer QoL) and 7 (no impact that is, best QoL). All outcome measures used are validated for children and previously used in our studies. ${ }^{28} 30$

\section{Sample size}

Our sample size estimates are based on our primary outcome (rate of acute doctor visits for respiratory exacerbations at 12 months). We aim to enrol 198 children with bronchiectasis. Our sample size is based on $30 \%$ improvement in the rate of non-scheduled doctor visits. Based on our current cohort data, the mean acute doctor visit rate is three per child year (SD 2.1). A $30 \%$ improvement equates to 2.1 per child year in the intervention arm. For $80 \%$ power and 5\% significance, we require 86 per group. To account for $15 \%$ dropout rate, our total required sample size is 198 (99 per group).

The sample size will provide power of $98 \%$ to detect the minimal important difference (0.6) between groups for PC-QoL at the different time frames and $95 \%$ power for a $50 \%$ improvement in influenza vaccine proportion (from current rate of $50 \%$ ). 
The sample size was calculated at application of the first grant (submitted September 2017) based on the exacerbation rate of children with bronchiectasis in Brisbane. This was before the current SARS-CoV-2 pandemic which has resulted in a substantial fall in acute respiratory exacerbations (our primary outcome). Thus, it is unknown but likely that our a priori calculated sample size will be inadequate, resulting in a type II error.

\section{Statistical analyses and reporting}

Data coding and entry is conducted in accordance with good clinical practice. Data will be reported and presented in accordance with the updated Consolidated Standards of Reporting Trials criteria. ${ }^{33}$ Children will be analysed according to the intention-to-treat principle where analysis will be by allocation status regardless of subsequent management. A detailed statistical analysis plan will be in place prior to data analyses. We will use a negative binomial regression model (as recommended, ${ }^{34}$ including treatment group, stratifying factors as independent factors, to determine between-group differences (with $95 \% \mathrm{CI}(\mathrm{CI})$ ), as done previously. ${ }^{27} 28$

For our secondary aims, the effects of the intervention will be determined by group differences in: (1) PC-QoL scores at 6 and 12 months and (2) overall respiratory exacerbation rate, will be analysed using ANCOVA (analysis of covariance) and presented as the mean difference $(95 \% \mathrm{CI})$. For the other secondary outcome, proportion with timely annual influenza vaccination will be compared between groups using $\chi^{2}$ tests to determine the OR with $95 \%$ CIs.

\section{Patient and public involvement}

Discussions with consumers, caregivers of children with bronchiectasis, in Darwin provided feedback on frustrations at not being able to access appropriate bronchiectasis management for their child, resulting in the preliminary ideas for a BAMP development. The study was subsequently discussed with, and approved by, the Menzies Child Health First Nations Reference Group (Darwin) prior to submitting the grant application. Also, the study was discussed with our CRE Parent and Community Advisory Group for bronchiectasis <https://www. crelungs.org.au/> who fully supported the BAMP and its design, study framework and outcome measures of the trial. Hence, although patients were not involved in the scientific study design, as mentioned above, they were involved in the choice of outcomes and overall approach.

\section{Ethics, dissemination and safety monitoring}

The Human Research Ethics Committees of all the recruiting institutions (Darwin: Northern Territory Department of Health and Menzies School of Health Research (2018-3081)) and (Brisbane, which covers Perth (HREC/18/QCHQ/45348)) have approved the RCT.

We will publish the results in a major medical journal (using the ICMJE author guidelines) and share the outcomes with the academic and medical community, funding and relevant patient organisations. Professional writers will not be used.

Study results will provide evidence for/against the use of BAMP when managing children with bronchiectasis. Data will be incorporated into future Cochrane reviews and national and local bronchiectasis guidelines. Project outcomes will be disseminated through the usual scientific manner (conferences and journals) as well as workshops, training programmes and guidelines the research team members are directly involved in and their networks. Dissemination will also include education workshops to health practitioners. The BAMP will be made freely available on our website of resources for clinicians/educators https://www.crelungs.org.au/. We anticipate our hospitals and outreach clinics (eg, to the Torres Strait and Indigenous Outreach Respiratory Care program ${ }^{35}$ ) will use them, as already expressed by the local clinical respiratory nurses. A First Nations Reference Group oversees the cultural aspects of the study. Given the nature of the study, we deemded that an independent data monitoring committee was unncessary.

\section{DISCUSSION}

We are currently undertaking a multicentre, double-blind RCT in three hospitals to address the question of whether providing an individualised BAMP to primary caregivers of children with bronchiectasis improves the clinical outcomes of non-scheduled doctor visits for respiratory exacerbations, overall exacerbation rate, PC-QoL and timely annual influenza vaccination.

\section{Rationale for our chosen outcome measures and timeframe}

Choosing outcomes that are both valid and consumerinformed are important for improving health. Respiratory exacerbation and PC-QoL as outcomes were chosen when developing our study plan as they were considered the most important from the consumer perspective. Also, published data show that respiratory exacerbations have major negative health impacts on people with underlying lung diseases. ${ }^{15} 16$ Thus, respiratory exacerbation rate reduction is our chosen primary outcome.

As PC-QoL is important from the patients' perspective, its inclusion as an outcome measure in an RCT is imperative. Our other secondary outcomes (overall respiratory exacerbation rate) are also important from both consumer and clinical perspectives, informed by the parent advisory group to our current ERS taskforce for guidelines on managing bronchiectasis in children. ${ }^{21}$ However, our sample size may limit power to detect a significant difference between groups for these other secondary outcomes.

Timely vaccinations are important for disease protection. ${ }^{36}$ Annual influenza vaccination is recommended in people with chronic lung disease and receiving the vaccine prior to the influenza season (ie, timely vaccination) can reduce respiratory exacerbations in children 
with bronchiectasis. Thus, our study is also evaluating timely annual influenza vaccination.

We chose evaluation at 12 months for several reasons: (1) our sample size calculation is based on our pilot data over the same timeframe; (2) evaluation over all four seasons is important as respiratory exacerbations are more common in winter; and (3) BAMPs are likely to be used long-term (ie, years) and not months. Thus, it is logical to undertake a study of at least 12 months duration.

In summary, our study addresses a clinical knowledge gap and consumer priority in the management of children with bronchiectasis, an increasingly appreciated chronic respiratory disease. The multicentre nature of our study increases the generalisability of the future findings of our RCT.

\author{
Author affiliations \\ ${ }^{1}$ Australian Centre for Health Services Innovation, Queensland University of \\ Technology, Brisbane, Queensland, Australia \\ ${ }^{2}$ Department of Respiratory and Sleep Medicine, Queensland Children's Hospital, \\ South Brisbane, Queensland, Australia \\ ${ }^{3}$ Child Health Division, Menzies School of Health Research, Charles Darwin \\ University, Darwin, Northern Territory, Australia \\ ${ }^{4}$ School of Medicine, University of Queensland, Brisbane, Queensland, Australia \\ ${ }^{5}$ College of Nursing and Midwifery, Charles Darwin University, Darwin, Northern \\ Territory, Australia
}

Acknowledgements We are grateful to members of the Menzies Child Health First Nations Reference Group at Menzies School of Health Research (Bilawara Lee, Mark Mayo, Tracey Brand, Christine Campbell, Dennis Bonney, Lorna Murakami-Gold, Veronica McClintic, Lesley Versteegh, Loyla Leysley) for supporting this study and for overseeing the cultural aspects.

Contributors $A B C$ conceived and designed the study, drafted the manuscript and was primarily responsible for obtaining the main grant from the Queensland Children's Hospital Foundation. JM codesigned the study, codrafted the manuscript and was the non-blinded doctor who wrote the final BAMP for each child at the start and on completion of the study. GBM codesigned the study, obtained the funding for Darwin, wrote the study protocol, designed the data collection forms, developed the database, obtained site specific ethics and governance for the Darwin site and coordinated all aspects of Perth as a new site. AC and JR coprepared the SOPs in Brisbane and oversaw the RCT in Brisbane. KS prepared the SOPs for Darwin and oversaw the RCT in Darwin with support from LV and PM who assisted with recruitment of participants. SY developed statistical plan for the study protocol. All authors read and approved the final manuscript.

Funding This work was supported by a project grant from the Queensland Children's Hospital Foundation (CHF), grant number 50273 and NHMRC HOT NORTH Centre for Research Excellence (APP1131932). ABC is supported by a NHMRC practitioner fellowship (grant APP1154302). JM is supported by a CHF early career fellowship (grant RPC0772019).

Disclaimer The views expressed in this publication are those of the authors and do not reflect the views of the funders.

Competing interests None declared.

Patient and public involvement Patients and/or the public were involved in the design, or conduct, or reporting, or dissemination plans of this research. Refer to the Methods section for further details.

Patient consent for publication Not applicable.

Provenance and peer review Not commissioned; externally peer reviewed.

Open access This is an open access article distributed in accordance with the Creative Commons Attribution Non Commercial (CC BY-NC 4.0) license, which permits others to distribute, remix, adapt, build upon this work non-commercially, and license their derivative works on different terms, provided the original work is properly cited, appropriate credit is given, any changes made indicated, and the use is non-commercial. See: http://creativecommons.org/licenses/by-nc/4.0/.
ORCID iD

Julie M Marchant http://orcid.org/0000-0002-8614-1829

\section{REFERENCES}

1 Chang AB, Bush A, Grimwood K. Bronchiectasis in children: diagnosis and treatment. Lancet 2018;392:866-79.

2 Chalmers JD, Chang AB, Chotirmall SH, et al. Bronchiectasis. Nat Rev Dis Primers 2018;4:45.

3 Chotirmall SH, Chalmers JD. Respire: breathing new life into bronchiectasis. Eur Respir J 2018;51:1702444.

4 Henkle E, Aksamit TR, Daley CL, et al. Us patient-centered research priorities and roadmap for bronchiectasis. Chest 2018;154:1016-23.

5 Chotirmall SH, McShane PJ. Time to acknowledge, address, and take action against bronchiectasis. Lancet Glob Health 2019;7:e1162-3.

6 Snell N, Gibson J, Jarrold I, et al. Epidemiology of bronchiectasis in the UK: Findings from the British lung foundation's 'Respiratory health of the nation' project. Respir Med 2019;158:21-3.

7 Seitz AE, Olivier KN, Adjemian J, et al. Trends in bronchiectasis among Medicare beneficiaries in the United States, 2000 to 2007. Chest 2012:142:432-9.

8 Munro KA, Reed PW, Joyce H, et al. Do New Zealand children with non-cystic fibrosis bronchiectasis show disease progression? Pediatr Pulmonol 2011;46:131-8.

9 Chang AB, Masel JP, Boyce NC, et al. Non-Cf bronchiectasis: clinical and HRCT evaluation. Pediatr Pulmonol 2003;35:477-83.

10 Goyal V, Grimwood K, Masters IB. State of the art: pediatric bronchiectasis. Pediatr Pulmonol 2016;51:450-69.

11 Chang AB, Robertson CF, Van Asperen PP, et al. A multicenter study on chronic cough in children : burden and etiologies based on a standardized management pathway. Chest 2012;142:943-50.

12 ERS. Bronchiectasis. In: The European lung white book., 2014: 15, 176-83.

13 Goyal V, McPhail SM, Hurley F, et al. Cost of hospitalization for bronchiectasis exacerbation in children. Respirology 2020;25:1250-6.

14 Prentice BJ, Wales S, Doumit M, et al. Children with bronchiectasis have poorer lung function than those with cystic fibrosis and do not receive the same standard of care. Pediatr Pulmonol 2019;54:1921-6.

15 Kapur N, Masters IB, Newcombe P, et al. The burden of disease in pediatric non-cystic fibrosis bronchiectasis. Chest 2012;141:1018-24

16 Bell SC, Elborn JS, Byrnes CA. Bronchiectasis: treatment decisions for pulmonary exacerbations and their prevention. Respirology 2018;23:1006-22.

17 Kapur N, Masters IB, Chang AB. Longitudinal growth and lung function in pediatric non-cystic fibrosis bronchiectasis: what influences lung function stability? Chest 2010;138:158-64.

18 Lovie-Toon YG, Grimwood K, Byrnes CA, et al. Health-resource use and quality of life in children with bronchiectasis: a multi-center pilot cohort study. BMC Health Serv Res 2019;19:561.

19 Goeminne PC, Hernandez F, Diel R, et al. The economic burden of bronchiectasis - known and unknown: a systematic review. BMC Pulm Med 2019;19:54.

20 Aliberti S, Masefield S, Polverino E, et al. Research priorities in bronchiectasis: a consensus statement from the EMBARC clinical research collaboration. Eur Respir J 2016;48:632-47.

21 Chang AB, Boyd J, Bell L, et al. Clinical and research priorities for children and young people with bronchiectasis: an international roadmap. ERJ Open Res 2021;7:00122-2021-2021.

22 GINA. Global strategy for asthma management and prevention. updated 2017. Global Initiative for Asthma, 2017.

23 Schutz KL, Marchant JM, Chang AB, et al. Perspective: using bronchiectasis action management plans for children with Bronchiectasis-Can it improve clinical care? Front Pediatr 2019;7:428.

24 Kelly C, Grundy S, Lynes D, et al. Self-Management for bronchiectasis. Cochrane Database Syst Rev 2018:2:Cd012528.

25 Newcombe PA, Sheffield JK, Chang AB. Parent cough-specific quality of life: development and validation of a short form. $J$ Allergy Clin Immunol 2013:131:1069-74.

26 Chan A-W, Tetzlaff JM, Gøtzsche PC, et al. Spirit 2013 explanation and elaboration: guidance for protocols of clinical trials. BMJ 2013;346:e7586.

27 Valery PC, Morris PS, Byrnes CA, et al. Long-Term azithromycin for Indigenous children with non-cystic-fibrosis bronchiectasis or chronic suppurative lung disease (bronchiectasis intervention study): 
a multicentre, double-blind, randomised controlled trial. Lancet Respir Med 2013;1:610-20.

28 Goyal V, Grimwood K, Byrnes CA, et al. Amoxicillin-Clavulanate versus azithromycin for respiratory exacerbations in children with bronchiectasis (BEST-2): a multicentre, double-blind, non-inferiority, randomised controlled trial. Lancet 2018;392:1197-206.

29 Chang AB, Robertson CF, van Asperen PP, et al. A cough algorithm for chronic cough in children: a multicenter, randomized controlled study. Pediatrics 2013;131:e1576-83.

30 Goyal V, Grimwood K, Ware RS. Efficacy of oral antibiotics for nonsevere exacerbations of bronchiectasis in children (best 1): a multicentre, double-blind, double-dummy, randomised placebo-controlled trial. Lancet Respir Med 2019;7:791-801.

31 Chang AB, Newman RG, Carlin JB, et al. Subjective scoring of cough in children: parent-completed vs child-completed diary cards vs an objective method. Eur Respir J 1998;11:462-6.
32 Kapur N, Masters IB, Morris PS, et al. Defining pulmonary exacerbation in children with non-cystic fibrosis bronchiectasis. Pediatr Pulmonol 2012;47:68-75.

33 Schulz KF, Altman DG, Moher D, et al. Consort 2010 statement: updated guidelines for reporting parallel group randomised trials. Trials 2010;11:32.

34 Keene ON, Jones MRK, Lane PW, et al. Analysis of exacerbation rates in asthma and chronic obstructive pulmonary disease: example from the TRISTAN study. Pharm Stat 2007;6:89-97.

35 Collaro AJ, Chang AB, Marchant JM, et al. Pediatric patients of outreach specialist Queensland clinics have lung function improvement comparable to that of tertiary pediatric patients. Chest 2020;158:1566-75.

36 Lovie-Toon YG, Hall KK, Chang AB, et al. Immunisation timeliness in a cohort of urban Aboriginal and Torres Strait Islander children. BMC Public Health 2016;16:1159. 